\title{
Large tunneling magnetoresistance effect at high voltage drop for Co-based Heusler alloy\#MgO\#CoFe junctions
}

\author{
A. D. Rata, H. Braak, D. E. Bürgler, and C. M. Schneider
}

Citation: Journal of Applied Physics 101, $09 J 503$ (2007);

View online: https://doi.org/10.1063/1.2711070

View Table of Contents: http://aip.scitation.org/toc/jap/101/9

Published by the American Institute of Physics

\section{Articles you may be interested in}

Influence of the crystal structure of thin Co films on X-ray magnetic linear dichroism-Comparison of ab initio theory and reflectometry experiments

Journal of Applied Physics 115, 17E132 (2014); 10.1063/1.4869292

Magnetoresistance response of cobalt nanocontacts between extended magnetic electrodes

Journal of Applied Physics 102, 083907 (2007); 10.1063/1.2798925

Spin-polarized photoemission spectroscopy of the $\mathrm{MgO} / \mathrm{Fe}$ interface on $\mathrm{GaAs}(100)$

Journal of Applied Physics 95, 7240 (2004); 10.1063/1.1669214

Soft x-ray longitudinal magneto-optical Kerr effect measured from $\mathrm{Co} / \mathrm{Cu}$ multilayers at the $\mathrm{CoL}_{2,3}$ edges Journal of Applied Physics 93, 6516 (2003); 10.1063/1.1555320

Interdiffusion, stress, and microstructure evolution during annealing in $\mathrm{Co} / \mathrm{Cu} / \mathrm{Co}$ trilayers Journal of Applied Physics 91, 9696 (2002); 10.1063/1.1479750

Dominant role of the size effect for saturation resistivity and giant magnetoresistance in Co/Cu multilayers Journal of Applied Physics 89, 7118 (2001); 10.1063/1.1359224

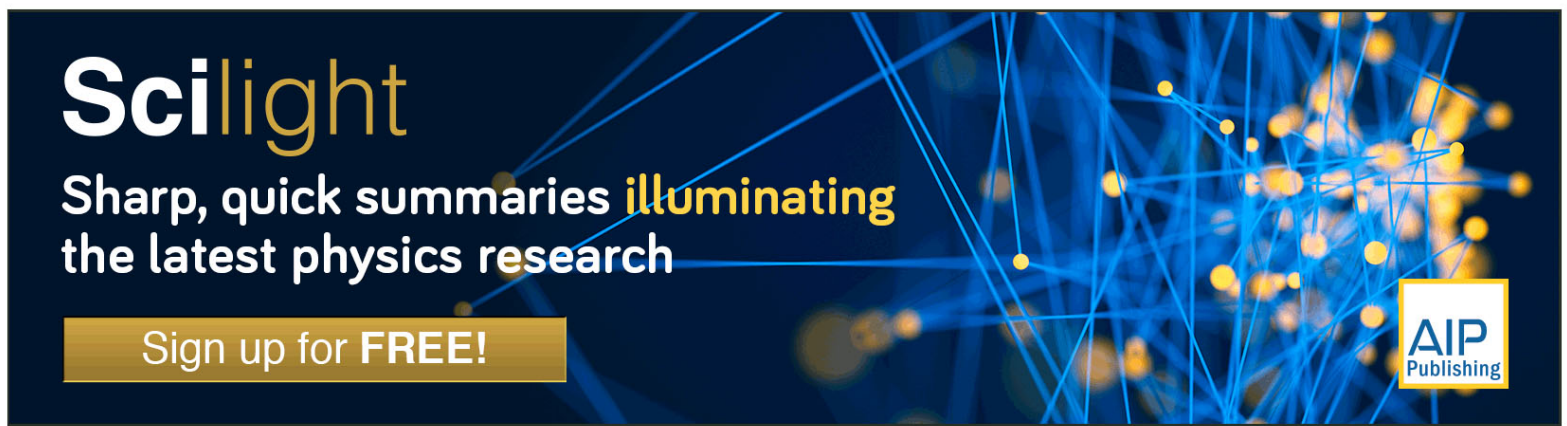




\title{
Large tunneling magnetoresistance effect at high voltage drop for Co- based Heusler alloy/MgO/CoFe junctions
}

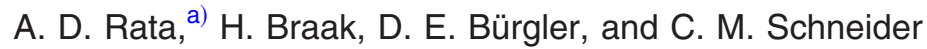 \\ Institute of Solid State Research, Electronic Properties (IFF-9) and cni-Center of Nanoelectronic Systems \\ for Information Technology, Research Center Jülich GmbH, D-52425 Jülich, Germany
}

(Presented on 11 January 2007; received 27 October 2006; accepted 11 December 2006; published online 5 April 2007)

\begin{abstract}
Growth and magnetic characterization of thin films of $\mathrm{Co}_{2} \mathrm{Cr}_{0.6} \mathrm{Fe}_{0.4} \mathrm{Al}$ and $\mathrm{Co}_{2} \mathrm{MnSi}$ full-Heusler compounds are investigated. Thin films were deposited by magnetron sputtering at room temperature directly onto oxidized Si wafers. These Heusler films are magnetically very soft and ferromagnetic with Curie temperatures well above room temperature. Polycrystalline $\mathrm{Co}_{2} \mathrm{Cr}_{0.6} \mathrm{Fe}_{0.4} \mathrm{Al}$ Heusler films combined with $\mathrm{MgO}$ barriers and $\mathrm{CoFe}$ counter electrodes are structured to magnetic tunnel junctions and yield almost 50\% magnetoresistance at room temperature. The magnetoresistance shows a strong bias dependence with the maximum occurring at a voltage drop well above $1 \mathrm{~V}$. This special feature is accompanied by only a moderate temperature dependence of the tunnel magnetoresistance. (C) 2007 American Institute of Physics.

[DOI: $10.1063 / 1.2711070]$
\end{abstract}

\section{INTRODUCTION}

Co-based Heusler alloys are predicted to belong to the material class of the half metals. ${ }^{1}$ They are characterized by a band gap at the Fermi energy for one spin direction and metallic properties for the other. Therefore, the charge carriers should be $100 \%$ spin polarized. Due to this feature large tunneling or giant magnetoresistance values are expected in layered structures incorporating Heusler alloys as one or both ferromagnetic electrodes.

In recent years, the Heusler compounds have been shown to yield increased tunneling magnetoresistance (TMR) values compared to the $3 d$-transition metals elements and their alloys. Still, the experimentally observed TMR values are way below the expectations. It is generally recognized that site disorder ${ }^{2}$ and the presence of interface states may drastically affect and even destroy the half metallicity. However, significant progress was recently made in improving the growth quality and reducing the site disorder. Thin films of Co-based Heusler alloys were successfully prepared in the ordered $L 2_{1}$ structure. $^{4-6}$ These films display improved magnetic characteristics with Curie temperatures and magnetic moments close to the bulk values. Moreover, theoretical calculations ${ }^{7}$ showed that Co-based full-Heusler alloys are more tolerant to site disorder, such that a high spin polarization is preserved even in the disordered $B 2$ structure. Among the Co-based full-Heusler compounds, $\mathrm{Co}_{2} \mathrm{Cr}_{0.6} \mathrm{Fe}_{0.4} \mathrm{Al}$ (CCFA) and $\mathrm{Co}_{2} \mathrm{MnSi}(\mathrm{CMS})$ are promising candidates for obtaining large tunneling or giant magnetoresistance effects. They have large magnetic moments and high Curie temperatures $\left(T_{C}\right)$, with $\mathrm{CMS}\left(T_{C} \approx 985 \mathrm{~K}\right)$ being among the highest. ${ }^{8}$ Several groups have successfully prepared thin films of CCFA and CMS on various substrates, showing improved structural order and magnetic characteristics (see, for example, Refs. ${ }^{4,6,9-11}$ ). Remarkable progress

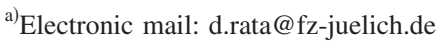

was obtained also by incorporating these compounds into magnetic tunnel junctions (MTJs). Fully epitaxial junctions with Heusler CCFA films and $\mathrm{MgO}$ tunneling barriers showed 90\% TMR ratio at room temperature (RT) and $240 \%$ at $4.2 \mathrm{~K}^{4}$ Presently, the largest TMR ratio for Heusler compounds is $570 \%$ at $2 \mathrm{~K}$. It was obtained in layered structures comprising CMS as both ferromagnetic electrodes and an amorphous $\mathrm{AlO}_{x}$ barrier. ${ }^{12}$

We investigate thin films of CCFA and CMS full-Heusler compounds grown by magnetron sputtering at RT. In this contribution, we discuss the magnetic characteristics of CCFA and CMS thin films deposited directly onto oxidized Si wafers and report on large TMR values obtained at RT in layered structures combining a polycrystalline CCFA thin film with a $\mathrm{MgO}$ tunnel barrier and a $\mathrm{CoFe}$ counter electrode. The magnetoresistance shows on the one hand a rather moderate temperature dependence and on the other hand a strong bias dependence with the largest TMR value occurring at a high voltage drop clearly exceeding $1 \mathrm{~V}$.

\section{EXPERIMENT}

CCFA and CMS thin films with a thickness of $25 \mathrm{~nm}$ as well as the MTJs were grown directly onto Si wafers covered with native, amorphous $\mathrm{SiO}_{2}$ by magnetron sputtering at $\mathrm{RT}$. After optimization, the sputtering Ar pressure was 1 $\times 10^{-3}$ mbar for CCFA films and $4.5 \times 10^{-3}$ mbar for CMS films, respectively, at a target-substrate distance of $5 \mathrm{~cm}$. We used stoichiometric $\mathrm{Co}_{2} \mathrm{Cr}_{0.6} \mathrm{Fe}_{0.4} \mathrm{Al}$ and $\mathrm{Co}_{2} \mathrm{MnSi}$ targets for depositing our films. The sputtering rates, measured with a quartz balance, were calibrated using x-ray reflectivity. After deposition, the Heusler films can be annealed in situ up to $873 \mathrm{~K}$. The structure of the Heusler films was identified $e x$ situ by $\mathrm{x}$-ray diffraction (XRD) carried out with a Philips $\mathrm{X}$ 'Pert MRD diffractometer using $\mathrm{Cu} K \alpha$ radiation. For the magnetic characterization, we employed superconducting quantum interference device (SQUID) magnetometry. Lay- 
ered structures are prepared without breaking the vacuum with the following layer sequence: $\mathrm{SiO}_{2} / \mathrm{CCFA}$ $(25 \mathrm{~nm}) / \mathrm{MgO}(3 \mathrm{~nm}) / \mathrm{CoFe}(5 \mathrm{~nm}) / \operatorname{IrMn}(15 \mathrm{~nm})$. The $\mathrm{MgO}$ barrier was deposited by rf-stimulated discharge from a stoichiometric target. Junctions with area from $3 \times 3$ up to $15 \times 15 \mu \mathrm{m}^{2}$ with crossed electrodes were patterned for magnetotransport measurements in the currentperpendicular-plane (CPP) geometry by standard optical lithography. The transport measurements were performed with a dc setup in the four-point geometry using a constant current source. As for the SQUID measurements, the magnetic field is always applied in the film plane.

\section{RESULTS AND DISCUSSION}

Ideally, the full-Heusler compounds display the cubic, so-called $L 2_{1}$ structure consisting of four interpenetrating fcc unit cells. Structural disorder occurs easily in this type of crystallographic structure. XRD (not shown) indicates the polycrystalline structure of our CCFA and CMS Heusler films deposited directly onto amorphous $\mathrm{SiO}_{2}$ at RT. The CCFA films show a uniform distribution of grains with an average diameter of $40 \mathrm{~nm}$ and a rms roughness of $0.5 \mathrm{~nm} .^{11}$ The films take the so-called $B 2$ structure ( $\mathrm{CsCl}$ type), where Co occupies the proper sublattice, while disorder occurs between the other elemental constituents. Postannealing has little influence on the crystallographic structure. More details about growth and structure characterization of CCFA films can be found in Ref. 11 .

Despite the low deposition temperature and the polycrystalline growth, the magnetic characteristics of these films are comparable with results reported in literature for films deposited at higher temperature. ${ }^{6,13,14}$ SQUID measurements of $25 \mathrm{~nm}$ thick CCFA and CMS films revealed ferromagnetic order with Curie temperatures well above RT. Annealing in vacuum at relatively high temperatures increases $T_{C}$ for both CCFA and CMS films. The Curie temperature of the CCFA film can be increased up to $630 \mathrm{~K}$ after annealing in vacuum at $773 \mathrm{~K}$ for $1 \mathrm{~h}$. In the case of CMS films, we performed annealing at $623 \mathrm{~K}$ for $1 \mathrm{~h}$. The evaluation of the temperature dependence of the saturation magnetization based on SQUID data taken at temperatures up to $450 \mathrm{~K}$ indicates a $T_{C}$ above $800 \mathrm{~K}$. However, high temperature measurements are necessary to estimate the Curie temperature of CMS films more precisely. Both CCFA and CMS Heusler films display a soft ferromagnetic behavior. In Fig. 1, we plot the normalized $M-H$ loops of $25 \mathrm{~nm}$ thick CCFA and CMS Heusler films, respectively, measured at RT. The coercive field $H_{c}$ of the CCFA film is about $6 \mathrm{mT}$. Therefore, our CCFA films are magnetically softer compared to reported results about CCFA films deposited on $\mathrm{Al}_{2} \mathrm{O}_{3}$ substrates at high temperatures, which have a coercive field of $10 \mathrm{mT}{ }^{15}$ Moreover, the CMS film are magnetically even softer having a $H_{c}$ of only $2 \mathrm{mT}$.

We estimated the total magnetic moments from the saturation magnetization at $5 \mathrm{~K}$. As expected, the CMS films have higher magnetic moment than the CCFA layers. We find that annealing in vacuum increases not only $T_{C}$ but also the value of the total magnetic moment. For the CCFA films we

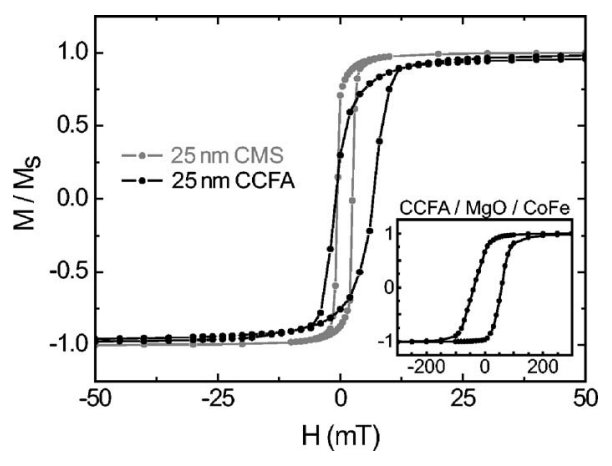

FIG. 1. Normalized magnetic hysteresis curves measured by SQUID at RT of $25 \mathrm{~nm}$ thick CCFA (black) and CMS (gray) films on $\mathrm{SiO}_{2}$. The CCFA and CMS films are ferromagnetically soft with a $H_{c}$ of 6 and $2 \mathrm{mT}$, respectively. The field biases are due to antiferromagnetic oxides that form on the surface of the uncapped films upon exposure to ambient air. Inset: magnetic hysteresis curve of a CCFA $(25 \mathrm{~nm}) / \mathrm{MgO}(3 \mathrm{~nm}) / \mathrm{CoFe}(5 \mathrm{~nm}) / \operatorname{IrMn}(15 \mathrm{~nm})$ extended layered structure measured before lithography at $77 \mathrm{~K}$.

obtained $2.56 \mu_{B}$ /f.u. and CMS films have a magnetic moment of $4.42 \mu_{B} /$ f.u. These values are lower than the predicted theoretical values, which are $3.8 \mu_{B}$ /f.u. for CCFA and $5 \mu_{B}$ /f.u. for CMS. These differences indicate the presence of site disorder in both materials. In the case of CCFA films, however, the value of the total magnetic moment is comparable with results obtained from films grown at elevated temperatures. ${ }^{13}$ On the other hand, the CMS films have a total magnetic moment close to the experimental bulk value of $5.07 \mu_{B}$ /f.u.. ${ }^{8}$ We take this as an indication for less atomic disorder in the CMS films in comparison with the CCFA layers.

Magnetic tunneling junctions were prepared from stacks with the layer sequence $\mathrm{SiO}_{2} / \mathrm{CCFA}(25 \mathrm{~nm}) / \mathrm{MgO}$ $(3 \mathrm{~nm}) / \mathrm{CoFe}(5 \mathrm{~nm}) / \mathrm{IrMn}(15 \mathrm{~nm})$. The completed structure is annealed in vacuum for $1 \mathrm{~h}$ at $573 \mathrm{~K}$. Although an IrMn antiferromagnet is located next to the upper CoFe electrode, the two ferromagnetic layers do not switch separately as observed in SQUID measurements performed on the extended multilayer before lithographically defining the MTJs (inset of Fig. 1). We mention at this point that no further treatment such as annealing above the Néel temperature of IrMn and field cooling could be applied to this structure. Thus, the CoFe electrode is not exchange biased. The bellshaped magnetoresistance curves without separate switching fields are consistent with the SQUID measurements and indicate the presence of magnetic coupling between CCFA and CoFe. Antiferromagnetic, Néel-type and biquadratic couplings due to interface roughness are the most likely coupling mechanisms. This suggests a noncollinear orientation of the layer magnetizations in the field free state and, thus, the observed TMR values seem to be a lower limit. The switching behavior is different from that of fully epitaxial $\mathrm{CCFA} / \mathrm{MgO} / \mathrm{CoFe} \mathrm{MTJ}$ with much lower roughness and thus negligibly small coupling reported by Marukame et al. ${ }^{4}$

In Fig. 2, we plot several magnetoresistance curves measured at RT with different bias currents $I_{\text {bias }}$. For this experiment, we used a constant current source and vary $I_{\text {bias }}$ in steps of $0.1 \mathrm{~mA}$. The TMR ratio is defined as TMR $=\left(R_{\mathrm{AP}}\right.$ $\left.-R_{\mathrm{P}}\right) / R_{\mathrm{P}}$, where $R_{\mathrm{AP}}$ and $R_{\mathrm{P}}$ are the resistances for the antiparallel and parallel magnetization configurations, respec- 


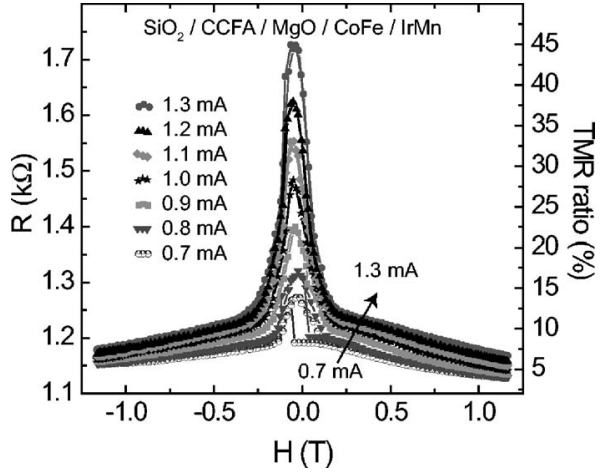

FIG. 2. Magnetoresistance curves of a $10 \times 10 \mu \mathrm{m}^{2}$ junction with layer sequence $\mathrm{SiO}_{2} / \mathrm{CCFA}(25 \mathrm{~nm}) / \mathrm{MgO}(3 \mathrm{~nm}) / \mathrm{CoFe}(5 \mathrm{~nm}) / \mathrm{IrMn}(15 \mathrm{~nm})$ measured by varying the bias current between 0.7 (bottommost curve) and $1.3 \mathrm{~mA}$ (topmost curve) in steps of $0.1 \mathrm{~mA}$. The measurements are performed at RT and yield a maximum magnetoresistance of $46 \%$. Joule heating at high currents might be the reason for the disappearing of hysteresis for currents larger than $0.9 \mathrm{~mA}$.

tively. We obtained a maximum TMR value of $46 \%$ for $I_{\text {bias }}=1.3 \mathrm{~mA}$. For smaller $I_{\text {bias }}$ the TMR ratio is much lower (about $1 \%$ for $I_{\text {bias }} \leqslant 0.6 \mathrm{~mA}$ ) and also for larger $I_{\text {bias }}$ the TMR ratio decreases, e.g., $18 \%$ for $I_{\text {bias }}=1.4 \mathrm{~mA}$ and $<7 \%$ for $I_{\text {bias }}=1.5 \mathrm{~mA}$. A variation of the bias current corresponds to a variation of the voltage drop across the junction. Multiplying the $I_{\text {bias }}$ values in Fig. 2 by the corresponding resistance values in the saturated state, we obtain voltage drops in the range between 0.81 and $1.53 \mathrm{~V}$. Therefore, the maximum TMR ratio occurs in these junctions at rather high voltages of more than $1 \mathrm{~V}$. For all data presented here, the CCFA electrode is at positive voltage with respect to the $\mathrm{CoFe}$ counter electrode. The minimal resistance-area product is about $100 \mathrm{k} \Omega \mu \mathrm{m}^{2}$. The measured junction resistances exceed $1 \mathrm{k} \Omega$ in all samples and, thus, are much larger than the lead resistances $(\approx 10-20 \Omega)$, indicating that there is no geometrical enhancement in the TMR ratios. The TMR values we obtained at RT are much larger compared to the results reported by Inomata et al. ${ }^{10}$ in similar structures also comprising a polycrystalline CCFA electrode.

In Fig. 3, finally, we present the temperature dependence of the TMR effect. A weak temperature dependence is observed with the TMR ratio increasing only by a factor of

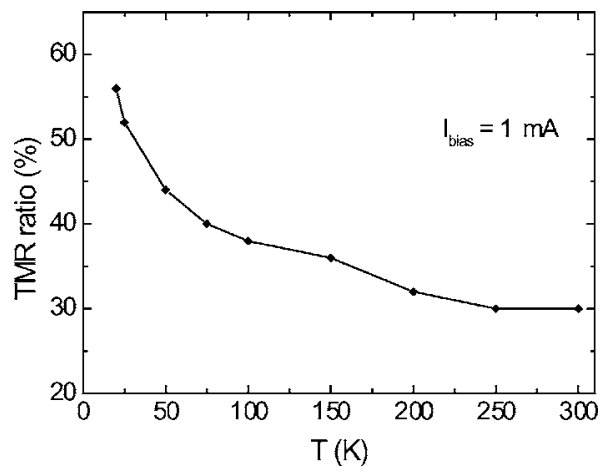

FIG. 3. Temperature dependence of the TMR ratio of a $10 \times 10 \mu \mathrm{m}^{2}$ junction with layer sequence $\mathrm{SiO}_{2} / \mathrm{CCFA}(25 \mathrm{~nm}) / \mathrm{MgO}(3 \mathrm{~nm}) /$ $\mathrm{CoFe}(5 \mathrm{~nm}) / \mathrm{IrMn}(15 \mathrm{~nm})$. The measurements are done with a constant bias current of $1 \mathrm{~mA}$. about 2 from $30 \%$ at RT to $56 \%$ at $20 \mathrm{~K}$. The measurements are performed with a constant bias current of $1 \mathrm{~mA}$. A similarly weak temperature dependence was observed by Yamamoto et al. ${ }^{16}$ in CCFA/MgO/CoFe structures, although the TMR values reported by these authors are slightly larger at low temperatures. The reason could be increased atomic order and better interfaces in their samples, which were prepared by molecular-beam epitaxy.

\section{CONCLUSIONS}

We prepared thin films of CCFA and CMS full-Heusler alloys by magnetron sputtering at room temperature. Our films are ferromagnetically soft, with coercive fields of about $6 \mathrm{mT}$ for CCFA and $2 \mathrm{mT}$ for CMS films. Annealing in vacuum increases the Curie temperature and the total magnetic moments. CCFA films combined with $\mathrm{MgO}$ barriers yield almost 50\% TMR at room temperature. Most surprising, the high TMR ratios occur only for high voltage drops across the junctions of up to $1.5 \mathrm{~V}$. The question whether this special magnetoresisitive behavior is related to spin filtering ${ }^{17}$ at the $\mathrm{MgO} / \mathrm{CoFe}$ interface of the "negative" electrode or to density-of-states effects in the "positive" CCFA electrode must be addressed in future experiments.

These results are encouraging for further investigations of TMR structures consisting of highly oriented full-Heusler alloy films combined with possibly epitaxial $\mathrm{MgO}$ tunneling barriers. From the application point of view, both the weak temperature dependence and the high voltage drop, which yields an output signal of $0.7 \mathrm{~V}$ at $I_{\text {bias }}=1.3 \mathrm{~mA}$, are of high relevance.

\section{ACKNOWLEDGMENTS}

We would like to thank P. Grünberg for valuable discussions and F.-J. Köhne for technical support.

${ }^{1}$ R. A. de Groot, F. M. Mueller, P. G. van Engen, and K. H. J. Buschow, Phys. Rev. Lett. 50, 2024 (1983).

${ }^{2}$ S. Picozzi, A. Contineza, and A. J. Freeman, Phys. Rev. B 69, 094423 (2004).

${ }^{3}$ I. Galanakis, J. Phys.: Condens. Matter 16, 3089 (2004).

${ }^{4}$ T. Marukame, T. Kasahara, K. Matsuda, T. Uemura, and M. Yamamoto, Jpn. J. Appl. Phys., Part 2 44, L521 (2005).

${ }^{5}$ Y. Sakuraba, J. Nakata, M. Oogane, H. Kubota, Y. Ando, A. Sakuma, and T. Miyazaki, Jpn. J. Appl. Phys., Part 2 44, L1100 (2005).

${ }^{6}$ J. Schmalhorst, S. Kämmerer, M. Sacher, G. Reiss, A. Hütten, and A. Scholl, Phys. Rev. B 70, 024426 (2004).

${ }^{7}$ Y. Miura, K. Nagao, and M. Shirai, Phys. Rev. B 69, 144413 (2004).

${ }^{8}$ Magnetic Properties of Metals, edited by H. P. J. Wijn (Springer, Berlin, 1991).

${ }^{9}$ D. D. Djayaprawira et al., Appl. Phys. Lett. 86, 092502 (2005).

${ }^{10}$ K. Inomata, S. Okamura, R. Goto, and N. Tezuka, Jpn. J. Appl. Phys., Part 2 42, L419 (2003).

${ }^{11}$ A. D. Rata, H. Braak, D. E. Bürgler, S. Cramm, and C. M. Schneider, Eur. Phys. J. B 52, 445 (2006).

${ }^{12}$ Y. Sakuraba, J. Nakata, M. Oogane, Y. Ando, H. Kato, A. Sakuma, T. Miyazaki, and H. Kubota, Appl. Phys. Lett. 88, 022503 (2006).

${ }^{13}$ R. Kelekar and B. M. Clemens, J. Appl. Phys. 96, 540 (2004).

${ }^{14}$ R. Kelekar and B. M. Clemens, Appl. Phys. Lett. 86, 232501 (2005).

${ }^{15}$ G. Jakob, F. Casper, V. Beaumont, S. Falk, N. Auth, H. J. Elmers, C. Felser, and H. Adrian, J. Magn. Magn. Mater. 290, 1104 (2005).

${ }^{16} \mathrm{M}$. Yamamoto, T. Marukame, T. Ishikawa, K. Matsuda, T. Uemura, and M. Arita, J. Phys. D 39, 824 (2006).

${ }^{17}$ W. H. Butler, X. G. Zhang, T. C. Schulthess, and J. M. Mc Laren, Phys. Rev. B 63, 054416 (2001). 